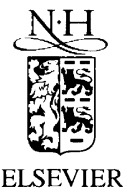

\title{
Regime jumps in electricity prices
}

\author{
Ronald Huisman*, Ronald Mahieu \\ Erasmus University Rotterdam, Room F4-18, P.O. Box 1738, Rotterdam 3000 DR, The Netherlands
}

\begin{abstract}
Many countries are liberalizing their energy markets. Participants in these markets are exposed to market risk due to the characteristics of electricity price dynamics. Electricity prices are known to be mean-reverting very volatile and subject to frequent spikes. Models that describe the dynamics of electricity prices should incorporate these characteristics. In order to capture the price spikes, many researchers have introduced stochastic jump processes, but we argue and show that this specification might lead to potential problems with specifying the true amount of mean-reversion within the process. In this paper, we propose a regimeswitching model that models price spikes separated from normal mean-reverting prices.
\end{abstract}

(c) 2003 Elsevier Science B.V. All rights reserved.

JEL classifications: $\mathrm{N70;}$ C51

Keywords: Energy markets; Electricity price modelling; Regime switching models; Spikes

\section{Introduction}

Participants in electricity markets face enormous market risks due to the highly volatile electricity markets. Daily volatilities of $29 \%$ are common; for comparison international stock indices have volatilities close to $20 \%$, but on a yearly basis. For risk management, portfolio management and option pricing issues it is crucial to have a good insight in the dynamics of electricity prices. Researchers have examined these dynamics and have indicated various stylised facts of electricity prices: high volatility, mean-reversion (prices tend to fluctuate around a long term equilibrium mean), seasonality (for example high summer prices in Arizona due to huge demand for power from air conditioning usage), and frequent extreme jumps in prices that

\footnotetext{
*Corresponding author. Tel.: +31-10-408-1450; fax: +31-10-408-9017.

E-mail addresses: r.huisman@fbk.eur.nl (R. Huisman), r.mahieu@ @bk.eur.nl (R. Mahieu).
} 
die out rapidly (result of fluctuations in demand and low elasticity of supply, due to system breakdown and limited inventory capacities). ${ }^{1}$

In this paper, we also focus on modelling electricity prices and concentrate on estimating the extreme jumps. Jumps in electricity prices are characterized by their short existence; prices fall back to a normal level sometimes after even one day (for example in case of system breakdown). The motivation for our study comes from past studies that have applied a stochastic jump model in combination with mean-reversion to model the jumps. The mean-reversion component is used to force the price of electricity to fall back to a normal level after a shock or jump has occurred; mean-reversion is directly associated with the jump process. However, it might well be that mean-reversion exists only in the 'normal' price process; the normal mean reverting process is then not specified correctly in traditional jump models. We argue that a stochastic jump process with mean-reversion might lead to an erroneous specification of the true mean-reversion process.

In this paper, we show the existence of such a normal mean reverting process that is not directly associated with jumps. In the regime jump model, we assume that the electricity price is in one out of three different regimes at each point in time. We identify a normal regime that can contain a mean-reversion component. In addition, we identify two extra regimes: the first regime models a price jump and a second regime models the way the process falls back to the normal process. Markov transition matrices specify the probabilities that the electricity prices move from one regime to another from one time point to the next. The advantage of the regime-switching framework is that we can explicitly model the short-lived characteristics of power spike, which is not captured by stochastic jump models.

Our results indicate that the electricity prices process exhibits significant meanreversion in its normal process and we show that the regime jump process performs better in modelling the jumps in combination with mean-reversion than a stochastic jump model. We therefore conclude that the regime jump model is a much richer specification of the electricity price dynamics than the other models used.

This paper is organized as follows. We explain the methodology in Section 2. In Section 3, we present summary statistics of the data we use. Section 4 shows empirical estimates from applying the model to electricity prices from several international markets. Section 5 concludes.

\section{Electricity price modeling}

Following Lucia and Schwartz (2002), we model the movement in the natural logarithm of the spot price for electricity as follows. Let $s(t)$ be the natural logarithm of the spot price at day $t$. We model the spot price as the sum of a deterministic component $f(\cdot)$ and a stochastic component $x(\cdot)$ :

$s(t)=f(t)+x(t), \quad$ where $t=1, \ldots, T$.

\footnotetext{
${ }^{1}$ See Pilipović (1998) and Clewlow and Strickland (2000) for results on stylised facts of electricity price dynamics.
} 
The component $f(\cdot)$ is a deterministic function of time and models the predictable regularities, such as any periodic behaviour and trends. We let $f(\cdot)$ account for the fact that prices for electricity delivered on weekend days is lower than the price on an average working day. To do so, let $D 1(t)$ be a dummy variable that equals 1 if $t$ is a Saturday and 0 for other days and $D 2(t)$ be a dummy that equals 1 if $t$ is a Sunday and 0 for other days. We specify $f(t)$ as follows:

$f(t)=\mu_{0}+\beta_{1} D 1(t)+\beta_{2} D 2(t)$

The parameter $\mu_{0}$ reflects the average price level. The second component $x(\cdot)$ in Eq. (1) is the stochastic component and can be interpreted as the spot price from which deterministic trends are removed. In this paper, we shall compare the impacts that spikes have on parameter estimates for different specifications of $x(t)$. We shall specify models that describe the change in the stochastic component of the spot price:

$d x(t)=d s(t)-d f(t), \quad$ where $t=1, \ldots, T$

The operator $d$ models the change in the value of the variable under consideration between today and the day before, i.e. $d x(t)=x(t)-x(t-1)$. In the following, we shall consider four models that describe the dynamics of $x(t)$ : a basic random walk type of model, a mean reverting model, a mean reverting model with stochastic jumps and a regime switching model.

\subsection{The basic model}

The basic model is a simplistic model in which we assume that the stochastic change in the spot price of electricity is normally distributed:

$d x(t)=\sigma_{0} \varepsilon(t), \quad$ where $\varepsilon(t) \sim N(0,1)$.

The parameter $\sigma_{0}$ is the volatility of the changes in the spot price.

\subsection{The mean-reverting model}

Previous studies ${ }^{2}$ have indicated that energy prices tend to move around the longterm equilibrium price level, modelled by $\mu_{0}$ in (Eq. (2)), which reflects the marginal costs of producing electricity. We introduce the rate of mean-reversion $\alpha_{0}$ that forces prices to move back to their long-term equilibrium value after the actual price has deviated from this equilibrium:

$d x(t)=-\alpha_{0} x(t-1)+\sigma_{0} \varepsilon(t), \quad$ where $\varepsilon(t) \sim N(0,1)$.

\footnotetext{
${ }^{2}$ See Pilipović (1998), Clewlow and Strickland (2000) and Lucia and Schwartz (2002) among others.
} 


\subsection{Incorporating spikes: stochastic jumps}

The existence of large jumps is another stylised fact of electricity prices. These spikes are often modelled by adding a Poisson error component to the meanreverting model Eq. (5). Let $Z(i)$ be the size of the $i$ th jump in the spot price and let $n$ be the actual number of jumps during the time interval under consideration, so $i=1, \ldots, n$. We assume that $Z$ is log-normally distributed. The mean-reverting model with Poisson jumps is given in the following equation:

$$
\begin{aligned}
d x(t)= & -\alpha_{0} x(t-1)+\lambda \sum_{i} \ln Z(i) \\
& +\sigma_{0} \varepsilon(t), \quad \text { where } \varepsilon(t) \sim N(0,1) \text { and } \ln Z(i) \sim N\left(\mu_{z}, \sigma_{z}^{2}\right)
\end{aligned}
$$

where $\lambda$ is the mean number of times that a jump occurs.

\subsection{Incorporating spikes: regime jumps}

The stochastic jump process used in the previous section allows for sudden jumps in the price level. This jump process is commonly used to model sudden level shifts in, for instance, stock prices. It does not explicitly model an important characteristic of electricity price spikes: spikes are temporal level shifts that die out rather quickly and do not lead to sustainable higher price levels. For example, a sharp price increase in one day due to system breakdown might lead directly to a jump down in prices on the following day when the system is repaired or when alternative supply is being generated. One may therefore expect that a sudden up-jump will be shortly followed by a down-jump or vice versa. The stochastic jump process introduced above only models a sustainable price increase, not explicitly the fast die-out property of electricity price jumps. Only in addition to a mean-reverting component in the price model as in Eq. (5), the stochastic jump process is capable of modelling the die-out property; mean-reversion forces extreme high or low prices to revert back to the long-term equilibrium price. However, this specification might lead to identification problems. If the price process in non-spike periods also exhibits mean-reversion, then the 'normal' mean-reverting process is calibrated with data from the jumps and could to an exaggeration of the amount of mean-reversion in normal periods.

In order to circumvent this problem, we introduce a regime-switching model that makes it possible to separately model the mean reversion in normal periods and spike periods. Following Hamilton and James (1989). ${ }^{3}$ To set up the regimes

\footnotetext{
${ }^{3}$ Hamilton and James (1989) introduced regime-switching models to describe exchange rate dynamics. Hamilton observed large swings in the value of the dollar relative to other currencies; long periods of dollar appreciation are followed with long periods of dollar depreciation. Obviously, the dynamics of the value of the dollar are different in each swing and Hamilton therefore introduced the regime model. In this model, the dollar is in one out of two regimes at each time period, representing an appreciation or a depreciation swing, and with a certain probability the dollar switches between both regimes from one period to another. Practically, regime models allow for distinct time series behaviour in each of the regimes.
} 
framework, we assume that on each day the electricity market can be in one out of three regimes: a normal regime (regime 0) when prices follow 'normal' electricity price dynamics, an initial jump regime (regime +1$)$ that models the process when the price of electricity suddenly increases or decreases in case of a spike and a regime (regime -1 ) that describes the process of how the electricity price reverts back to the normal regime after the spike has occurred. Since the occurrence of a jump is stochastic we capture the regimes in the specification for the stochastic component $d x(t)$ :

$d x(t)=-\alpha_{0} x(t-1)+\sigma_{0} \varepsilon(t), \quad$ in regime 0 with $\varepsilon(t) \sim N(0,1)$,

$d x(t)=\mu_{1}+\sigma_{1} \varepsilon(t), \quad$ in regime +1 with $\varepsilon(t) \sim N(0,1)$,

and

$d x(t)=-\alpha_{-1} x(t-1)+\sigma_{-1} \varepsilon(t)$, in regime -1 with $\varepsilon(t) \sim N(0,1)$

Note that when we condition on the regimes, the parameters of the model can easily be estimated by maximum likelihood. In order to estimate the regime dynamics we need to specify the mechanism that describes how to move from one regime to another. In regime switching models this is accomplished through a Markov transition matrix, which contains probabilities of jumping from a regime on a specific day to another regime on the next day. Maximum likelihood estimates of the parameters and the regimes can be found by applying Kalman filter methodology. ${ }^{4}$

\subsubsection{The Markov transition probabilities}

The switches between the regimes are controlled by one-period transition probabilities. Let $\pi(i, j)$ be the probability that the electricity price process switches from regime $j$ in period $t$ to regime $i$ in period $t+1$. We restrict the probabilities as follows. At any normal day, there is a probability of a spike tomorrow. Therefore, $\pi(0,0)$ equals the probability that no spike will occur and $\pi(+1,0)=1-\pi(0,0)$ is the probability of a spike. As we cannot proceed from a normal regime to the spike reverting regime we set $\pi(-1,0)$ equal to 0 . Being in a spike regime at time $t$, we assume that the reverting regime will start tomorrow, thus $\pi(-1,+1)$ equals 1 and $\pi(0,+1)$ and $\pi(+1,+1)$ are zero. Note that we assume that spikes last for only one day in this paper. This assumption can be relaxed by allowing different values for $\pi(-1,+1)$. Being in the reverting regime -1 at day $t$, we assume that we will be back in the normal regime tomorrow, thus $\pi(0,-1)$ equals 1 and $\pi(+1,-1)$ and $\pi(-1,-1)$ are zero. Again, this assumption can be relaxed by allowing for different values for $\pi(0,-1)$.

Given the assumptions outlined above, we only need to estimate the Markov

\footnotetext{
${ }^{4}$ See for example Harvey (1989).
} 
Table 1

\begin{tabular}{lccc}
\hline & APX & LPX & UK \\
\hline Start & Jan 2, 01 & Jun 15, 00 & Aug 15, 00 \\
End & Nov 5, 02 & Nov 5, 02 & Nov 5, 02 \\
$n$ & 673 & 865 & 786 \\
Mean & 0.001 & 0.000 & -0.001 \\
Stddev & 0.470 & 0.330 & 0.183 \\
Min & -2.374 & -1.425 & -1.217 \\
Max & 2.227 & 2.243 & 1.103 \\
Skew & 0.499 & 1.027 & 0.779 \\
Kurt $^{*}$ & 3.982 & 3.391 & 8.132 \\
\hline
\end{tabular}

Note: Start/End are the start and end dates of the series; $n$ is the number of observations.

${ }^{*}$ Kurt is excess kurtosis.

probability $\pi(0,0)$. In order to keep the estimates between 0 and 1 , we model the probability as follows:

$\pi(0,0)=\exp (p) /(1+\exp (p))$.

Therefore, we estimate the parameter $p$ and transform this into the probability according to Eq. (10).

\section{Data}

The electricity price data that we use in this study are day-ahead base load prices for the Dutch APX market, the German LPX market and the UK market (Telerate UK Power Index). All series are at a daily frequency. The sample lengths differ, however, depending on market existence and on the general availability of the data. The following table provides the summary statistics of the daily changes in natural logarithm of the prices.

From Table 1, we clearly observe the impacts of mean-reversion and spikes on the price dynamics: high daily volatility levels, positive skewedness and large excess kurtosis. The positive skewedness might indicate that the upward jumps are more intense than the jump reversals.

\section{Empirical results}

In this section, we compare the estimation results from the models that we described in Section 2. All parameters are estimated using maximum likelihood. Table 2 presents the estimates for the basic model Eq. (4). The average log price levels range from 2.9 for the UK through 3.4 for the APX. Daily volatility ranges from 0.2 for UK through 0.5 for the APX. Furthermore, a significant weekend effect is visible from the negative estimates for both $\beta$ coefficients. For all series, Sunday prices are lower than Saturday.

The basic model does not capture mean-reversion being one of the most important characteristics of power prices. Table 3 contains the parameter estimates for basic 
Table 2

Estimation results: basic model Eq. (4)

\begin{tabular}{llll}
\hline & APX & LPX & UK \\
\hline$\mu_{0}$ & 3.442 & 3.153 & 2.904 \\
& $(0.047)$ & $(0.034)$ & $(0.050)$ \\
$\mathrm{EqPr}^{*}$ & 31.259 & 22.422 & 18.253 \\
$\beta_{1}$ & -0.262 & -0.256 & -0.114 \\
& $(0.044)$ & $(0.022)$ & $(0.016)$ \\
$\beta_{2}$ & -0.625 & -0.535 & -0.227 \\
$\sigma_{0}$ & $(0.045)$ & $(0.023)$ & $(0.017)$ \\
LogLik & 0.524 & 0.308 & 0.215 \\
& $(0.021)$ & $(0.010)$ & $(0.009)$ \\
\hline
\end{tabular}

${ }^{*}$ EqPr: equilibrium price level. Standard errors are between parentheses.

model extended with mean-reversion Eq. (5). The log likelihood for the meanreversion model is lower than for the basic model presented in Table 2, indicating an improved fit. The mean-reversion parameter $\alpha$ is significant and positive for all power series and ranges from 0.21 for the UK series through 0.47 for the APX. This highlights the importance of mean-reversion in electricity price processes. Note also the impact from including mean-reversion on volatility estimates.

Note that the inclusion of mean-reversion leads to a richer specification of the electricity price process indicated by the lower log-likelihood values. Furthermore, imposing mean-reversion leads to a slightly reduction in volatility estimates. The effect is most noteworthy for the APX. The volatility estimate reduces from 0.52 to 0.37 due to including mean-reversion.

For both the basic model and the basic model with mean-reversion, spikes are implicitly modelled through volatility. Table 4 lists the parameter estimates for

Table 3

Estimation results: mean reverting model Eq. (5)

\begin{tabular}{llll}
\hline & APX & LPX & UK \\
\hline$\mu_{0}$ & 3.446 & 3.156 & 2.903 \\
EqPr $^{*}$ & $(0.050)$ & $(0.035)$ & $(0.049)$ \\
$\beta_{1}$ & 30.945 & 23.477 & 18.227 \\
$\beta_{2}$ & -0.267 & -0.259 & -0.115 \\
& $(0.047)$ & $(0.025)$ & $(0.017)$ \\
$\alpha$ & -0.627 & -0.538 & -0.227 \\
& $(0.045)$ & $(0.024)$ & $(0.018)$ \\
$\sigma_{0}$ & 0.473 & 0.284 & 0.206 \\
& $(0.018)$ & $(0.010)$ & $(0.008)$ \\
LogLik & 0.371 & 0.291 & 0.154 \\
\hline
\end{tabular}

${ }^{*}$ EqPr: equilibrium price level. Standard errors are between parentheses. 
Table 4

Estimation results: stochastic jump model Eq. (6)

\begin{tabular}{llll}
\hline & APX & LPX & UK \\
\hline$\mu_{0}$ & 3.283 & 3.144 & 2.775 \\
& $(0.026)$ & $(0.024)$ & $(0.037)$ \\
$\mathrm{EqPr}^{*}$ & 26.645 & 23.197 & 16.039 \\
$\beta_{1}$ & -0.236 & -0.253 & -0.056 \\
$\beta_{2}$ & $(0.021)$ & $(0.013)$ & $(0.007)$ \\
& -0.516 & -0.518 & -0.133 \\
$\alpha$ & $(0.026)$ & $(0.013)$ & $(0.009)$ \\
& 0.453 & 0.240 & 0.089 \\
$\sigma_{0}$ & $(0.032)$ & $(0.022)$ & $(0.014)$ \\
& 0.147 & 0.128 & 0.058 \\
$\sqrt{ } \lambda$ & $(0.016)$ & $(0.006)$ & $(0.004)$ \\
& 0.679 & 0.477 & 0.577 \\
$\lambda$ & $(0.083)$ & $(0.056)$ & $(0.042)$ \\
$\mu_{Z}$ & 0.461 & 0.228 & 0.333 \\
& 0.140 & 0.009 & 0.028 \\
$\sigma_{Z}$ & $(0.039)$ & $(0.0 .31)$ & $(0.014)$ \\
LogLik & 0.414 & 0.318 & 0.236 \\
& $(0.047)$ & $(0.036)$ & $(0.019)$ \\
& -109.882 & -252.053 & -582.291 \\
\hline
\end{tabular}

*EqPr: equilibrium price level. Standard errors are between parentheses.

model Eq. (6), which is an extension of the previous model. It allows both for mean-reversion and stochastic jumps in prices.

From Table 4 we infer that the frequency with which spikes occur in the market is very high. The $\lambda$ parameter, which equals the frequency of a jump in a stochastic jump framework, equals 0.46 for the APX. This would imply that more than $40 \%$ of all observations are seen as jumps. For the LPX market, the frequency is more than $20 \%$ and for the UK the frequency is 33\%. These frequencies are rather unlikely; we would expect values of $10 \%$ and lower. Compared with the results from the mean-reverting model in Table 3, we see that the volatility estimate has reduced in all cases by more than one half due to the inclusion of stochastic jumps. The fact the stochastic jump process identifies too much jumps, results in less volatility for the non-jump markets. This is especially apparent in the UK market, where the inclusion of stochastic jumps leads to a big reduction in the estimate of the mean-reversion parameter from 0.21 to 0.06 . These peculiar results are due to the fact that stochastic jumps are suited to model shifts to sustainable higher or lower price levels (for example in equity) markets, but not for non-sustainable price increases such as jumps that only last for a short period of time after which they revert back to normal levels. There is no component in the stochastic jump framework that allows the spike to last only for a short period of time. This difference in specification might explain the results we found. The short-life characteristic of power spikes is explicitly modelled in the regime-switching model.

Table 5 presents the parameter estimates for the regime-switching spike model Eqs. (7)-(9). The probability of a spike is low for all series. The parameter $\pi(0,0)$, 
Table 5

Estimation results: regime-switching model Eqs. (7)-(9)

\begin{tabular}{llll}
\hline & APX & LPX & UK \\
\hline$\mu_{0}$ & 3.368 & 3.146 & 2.852 \\
& $(0.042)$ & $(0.031)$ & $(0.045)$ \\
$\mathrm{EqPr}^{*}$ & 29.015 & 23.243 & 17.316 \\
$\mu_{1}$ & 0.377 & 0.037 & 0.103 \\
& $(0.184)$ & $(0.154)$ & $(0.097)$ \\
$\beta_{1}$ & -0.258 & -0.256 & -0.089 \\
& $(0.039)$ & $(0.022)$ & $(0.012)$ \\
$\beta_{2}$ & -0.559 & -0.532 & -0.192 \\
& $(0.040)$ & $(0.022)$ & $(0.014)$ \\
$\alpha_{0}$ & 0.371 & 0.299 & 0.112 \\
& $(0.041)$ & $(0.036)$ & $(0.026)$ \\
$\alpha_{-1}$ & 0.444 & 0.401 & 0.313 \\
& $(0.184)$ & $(0.157)$ & $(0.148)$ \\
$\sigma_{0}$ & 0.340 & 0.241 & 0.144 \\
& $(0.022)$ & $(0.015)$ & $(0.007)$ \\
$\sigma_{1}$ & 0.874 & 0.705 & 0.542 \\
& $(0.184)$ & $(0.194)$ & $(0.105)$ \\
$\sigma_{-1}$ & 0.918 & 0.477 & 0.453 \\
$P$ & $(0.211)$ & $(0.128)$ & $(0.092)$ \\
& 2.533 & 3.286 & 2.947 \\
$\pi(0,0)$ & $(0.470)$ & $(0.760)$ & $(0.331)$ \\
LogLik & 0.926 & 0.964 & 0.950 \\
\hline & -510.152 & -447.834 & -256.595 \\
\hline
\end{tabular}

${ }^{*}$ EqPr: equilibrium price level. Standard errors are between parentheses.

the probability that (while being in the normal regime today) the process will again be in the normal regime tomorrow, ranges from 0.92 for the APX through 0.96 for the LPX. The probability of a spike therefore, ranges from $8 \%$ to $4 \%$. The average size of a price jump $\mu_{1}$ is rather low, ranging from 0.04 for LPX through 0.38 for APX, but the level of expected spikes is very uncertain with standard errors ranging from 0.10 for UK through 0.18 for APX. The mean-reversion parameter for the spike reverting regime ranges from 0.31 (UK) through 0.44 (APX), which implies that prices strongly move back to normal levels after one day. Note that this estimate is influenced by our assumption that spikes only last for one day. Further research could experiment with more relaxed assumptions on the spike reverting process. The other parameters seem to be very robust with respect to the estimates from the mean-reverting model as presented in Table 3. The volatility estimates are somewhat reduced due to the fact that spikes are not captured through volatility in the regimeswitching model.

\section{Concluding remarks}

In this paper, we introduced a regime-switching framework to capture spikes in modelling the dynamics of power prices. Previous studies used stochastic jump 
process to model spikes, but these models do not account for the fact that power spikes are rather short-lived. Furthermore, the force with which the prices process reverts back to the long-rum price level is stronger after spikes than in normal periods. The methodology we proposed allows for different mean-reverting behaviour for normal periods and periods in which spikes occur. The parameter estimates indicate that the regime jump model is a better specification for simultaneously modelling mean-reversion and spikes in the dynamics of power prices.

\section{Acknowledgments}

All errors pertain to the authors. We thank Derek Bunn and participants to the ERIM research seminar, the EPRM 2001 conference and the Erasmus/Energy forum conference 2003 for helpful comments. Both authors are affiliated at the Erasmus University Rotterdam and at Energy Global Rotterdam.

\section{References}

Clewlow, L., Strickland, C., 2000. Energy Derivatives: Pricing and Risk Management. Lacima Publications.

Hamilton, , James, D., 1989. A new approach to the economic analysis of nonstationary time series and the business cycle. Econometrica 57, 357-384.

Harvey, A.C., 1989. Forecasting, Structural Time Series and the Kalman Filter. Cambridge University Press.

Lucia, J., Schwartz, E.S., 2002. Electricity prices and power derivatives: evidence from the Nordic power exchange. Rev. Derivatives Res. 5, 5-50.

Pilipović, D., 1998. Energy Risk: Valuing and Managing Energy Derivatives. McGrawHill Publishers. 